MATEC Web of Conferences 1, 10003 (2012)

DOI: $10.1051 /$ matecconf $/ 20120110003$

(C) Owned by the authors, published by EDP Sciences, 2012

\title{
Large amplitude free vibration analysis of functionally graded beams using an homogenisation procedure
}

\author{
K. EL Bikri ${ }^{1}$, A. Zerkane ${ }^{1}$ and R. Benamar ${ }^{2}$ \\ ${ }^{1}$ ENSET-Rabat, Mechanical Engineering Department, LaMIPI, B.P. 6207, Rabat InstitutsUniversité Mohammed V- \\ Souissi, Rabat, Morocco \\ ${ }^{2}$ EMI, EGT, LERSIM, Agdal Université Mohammed V-Souissi, Rabat, Morocco
}

\begin{abstract}
The purpose of the present paper is to show that the problem of geometrically non linear free vibrations of functionally graded (FG) beams with immovable ends can be reduced to that of isotropic homogeneous beams with effective bending stiffness and axial stiffness parameters. The material properties of the functionally graded composites examined are assumed to be graded in the thickness direction and estimated through the rule of mixture. The theoretical model is based on the Euler-Bernouilli beam theory and the Von Kármán geometrical nonlinearity assumptions. An homogenization procedure is developed using the governing axial equation of the beam in which the axial inertia and damping are ignored. Hamilton's principle is applied and a multimode approach is derived to calculate the fundamental nonlinear frequency parameters, which are found to be in a good agreement with the published results. The non-dimensional curvatures associated to the nonlinear fundamental mode are also given in the case of clamped-clamped FG beams.
\end{abstract}

\section{Introduction}

Recently, the developments in materials engineering have led to consideration of special composites called "functionally graded materials" (FGMs). They are characterized by smooth and continuous variations in their thermomechanical properties along the thickness of a structure obtained by gradually varying the volume fraction of the constituent materials. FGMs possess various advantages over the conventional composite laminates, such as smaller thermal stresses and stress concentrations and they can be designed to achieve specific properties for different applications. Indeed, FGMs are generally made of a mixture of ceramic and metal to satisfy the demand of ultra-high-temperature environment and to eliminate the interface problems. Hence, this new kind of materials has been employed in the design of many engineering structures such as aircrafts, space vehicles, defence industries, electronics and biomedical equipments.

Because of the wide applications of FGMs, it is important to study the dynamic behaviour of FGM structures such as beams which are used extensively as structural members and often subjected to relatively large vibration amplitudes, compared to their thickness. Consequently, many structural problems are encountered in these severe work conditions due to material fatigue which is accelerated leading to structural damages and catastrophic failures. These phenomena are more significant around the natural frequencies of the structure, for which the nonlinear vibration analysis becomes essential for a reliable structural design.

Recently, the dynamic response of FG beams has been the purpose of many research works. In [1], fundamental frequency analysis of FG beams having different boundary conditions has been made within the framework of the classical, the first-order and different higher-order shear deformation beam theories. Exact solutions for the bending vibration problem of FG beams with variation of material properties in a one-layer beam or in the layers of a multilayered sandwich beam have been proposed in [2]. The linear beam theory has been used for establishing the equilibrium and kinematical equations, taking into account the effect of the shear deformation and the effect of consistent mass distribution and mass inertia moment. In [3], the dynamic characteristics of a functionally graded beam with axially or transversally material graduation through the thickness have been presented. The finite element method has been employed under the assumptions of the Euler-Bernoulli beam theory to develop the discretized model and obtain a numerical approximation of the motion equation. A mixed method for forced vibration of functionally graded beams subjected to moving loads has been developed in [4]. The theoretical formulation is based on the EulerBernoulli beam theory, and the governing equations of 
motion of the system have been derived using Lagrange's equations. Then, the Rayleigh-Ritz method has been employed to discretize the spatial partial derivatives and the differential quadrature method has been used for the discretization of the temporal derivatives. Static and free vibration analysis of functionally graded layered beams have been conducted in [5] using a third order zigzag theory based model. Two systems, Al/SiC and Ni/Al2O3, fabricated using powder metallurgy and thermal spraying techniques respectively, have been considered for the experimental validation. The detection of cracks in structural members made of a functionally graded material has been a significant subject due to their increasing applications in various important engineering industries. A model-based approach has been developed in [6] to determine the location and size of an open edge crack in an FGM beam. The p- version of finite element method has been employed to estimate the transverse vibration characteristics of a cracked FG beam. In [7], approximate analytical expressions have been presented for geometrically nonlinear vibration and post-buckling analysis of FG beams on a nonlinear elastic foundation subjected to an axial force. The Euler-Bernoulli assumptions together with The Von Kaman's strain displacement relation have been employed to derive the governing partial differential equation of motion, the analytical solution of which has been obtained using one parameter Galerkin solution in conjunction with a variational approach.

In the present paper, the problem of geometrically non linear free vibrations of clamped-clamped FG beams with immovable ends is investigated using Hamilton's principle and spectral analysis. Based on the governing axial equation of the beam in which the axial inertia and damping are ignored, an homogenisation procedure is proposed which reduces the problem studied to that of isotropic homogeneous beams with effective bending stiffness and axial stiffness parameters.

\section{Functionally graded materials}

In this section, we consider a clamped-clamped FG beam having the geometrical characteristics shown in Fig. 1. It is assumed that the FG beam is made of ceramic and metal, and the effective material properties of the FG beam, i.e., Young's modulus $\mathrm{E}$ and mass density $\rho$, are functionally graded in the thickness direction according to a function of the volume fractions $\mathrm{V}$ of the constituents.

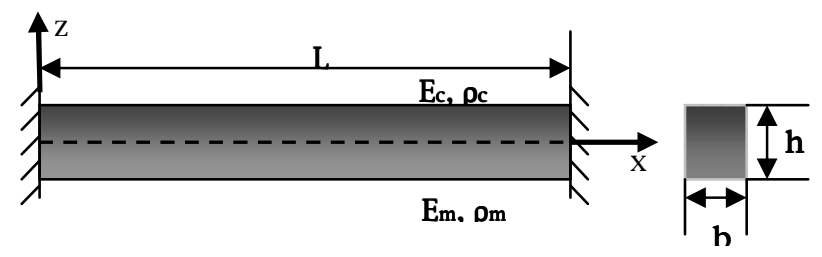

Fig. 1. FG beam notation.

According to the rule of mixture, the effective material properties $\mathrm{P}$, can be expressed as:
$P=P_{m} V_{m}+P_{c} V_{c}$

where subscripts " $\mathrm{m}$ " and "c" refer to the metal and ceramic constituents, respectively.

Various types of functions are used in the literature to describe the variation of the volume fraction of the constituents. Here, a simple power law is considered to describe the variation of the volume fraction of the metal and the ceramic constituents as follows:

$V_{m}=\left(\frac{z}{h}+\frac{h}{2}\right)^{k}$

with $V_{m}+V_{c}=1$

$\mathrm{k}$ is a non-negative parameter (power-law exponent) which dictates the material variation profile through the thickness of the beam.

Effective material properties of the FG beam such as Young's modulus (E) and mass density ( $\rho)$ can be determined by substituting Eq. (2) into Eq. (1), which gives:

$$
\begin{aligned}
& E(z)=E_{m}+\left(E_{c}-E_{m}\right)\left(\frac{z}{h}+\frac{1}{2}\right)^{k} \\
& \rho(z)=\rho_{m}+\left(\rho_{c}-\rho_{m}\right)\left(\frac{z}{h}+\frac{1}{2}\right)^{k}
\end{aligned}
$$

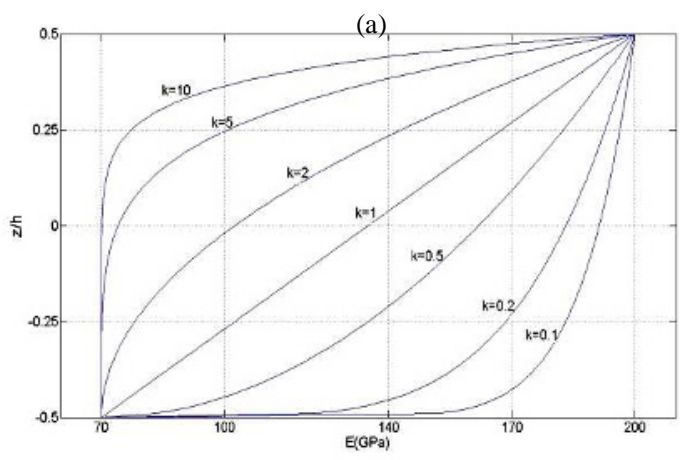

(b)

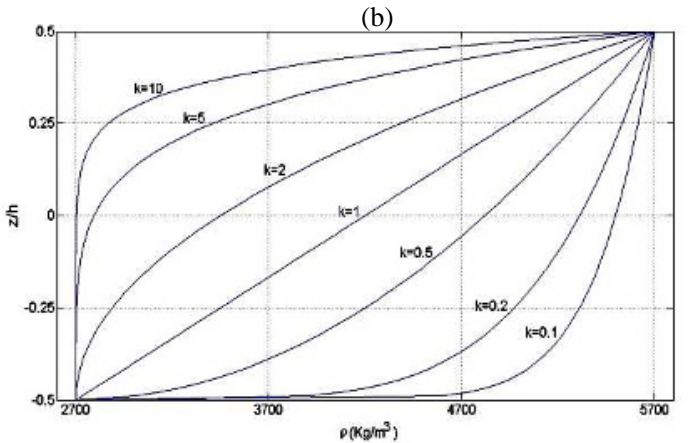

Fig. 2. Variation of the material properties of the FG beam through the thickness: (a) Young Modulus, (b) mass density

\section{Non linear free vibration analysis}

The Von Kármán type nonlinear strain-displacement relationships are given by: 
$\varepsilon_{x}^{a}=\frac{\partial u^{a}}{\partial x}+\frac{1}{2}\left(\frac{\partial W^{a}}{\partial x}\right)^{2}$

$K_{x}=\frac{\partial^{2} W^{a}}{\partial x^{2}}$

Where $\varepsilon_{x}^{a}$ is the non-linear axial strain, $K_{x}$ is the curvature of the beam. The total elastic strain energy $V_{T}$ of Euler-Bernoulli beams is given by:

$V_{T}=\frac{1}{2} \int_{0}^{l} N_{x} \varepsilon_{x}^{a}+M_{y} K_{x} d x$

In which $N_{x}$ and $M_{y}$ are the axial internal force and the bending moment respectively, which are related to the strains as follows:

$N_{x}=b A_{11} \varepsilon_{x}^{a}+b B_{11} K_{x}$

$M_{y}=b B_{11} \varepsilon_{x}^{a}+b D_{11} K_{x}$

Where $A_{11}, B_{11}$ and $D_{11}$ are the extension-extension, bending-extension, bending-bending coupling coefficients respectively, and can be evaluated using the classical laminate plate theory.

Using the static axial equilibrium equation for the beam, once the axial inertia and damping terms are neglected, and applying the immovable ends conditions, the potential energy $V_{T}$ can be written in terms of the transverse displacement $W^{a}$ as follows:

$V_{T}=\frac{(E S)_{e f f}}{8 l}\left(\int_{0}^{l}\left(\frac{\partial W^{a}}{\partial x}\right)^{2} d x\right)^{2}+\frac{(E I)_{e f f}}{2} \int_{0}^{l}\left(\frac{\partial^{2} W^{a}}{\partial x^{2}}\right)^{2} d x$

Where $(E S)_{e f f}=b A_{11}$ and $(E I)_{e f f}=b\left(D_{11}+\left(B_{11}^{2} / A_{11}\right)\right)$

are the effective axial and bending stiffness respectively. Expression (10) for the total strain energy is effective for replacing the FGM beam problem with an equivalent classical isotropic beam problem [8].

The kinetic energy, in which the axial and rotary inertia are ignored, is given by:

$T=\frac{1}{2} \int_{0}^{l} \int_{S} \rho(z)\left(\frac{\partial W^{a}}{\partial t}\right)^{2} d S d x$

For a general parametric study, we use the following non dimensional formulation by putting:

$x^{*}=\frac{x}{l}, W^{a^{*}}=\frac{W^{a}}{r}$, where $r=\sqrt{\frac{I}{S}}$

Applying Hamilton's principle and expanding the displacement $W^{a}$ in the form of a finite series, the following set of non linear amplitude equations is obtained:

$(3 / 2) a_{i} a_{j} a_{r} b_{i j k r}^{*}+a_{i} k_{i r}^{*}-\omega^{* 2} a_{i} m_{i r}^{*}=0, \mathrm{r}=1 \ldots \mathrm{n} .(12)$

$k_{i j}^{*}, b_{i j k l}^{*}$ and $m_{i j}^{*}$ stand for the non dimensional classical rigidity tensor, the non linear rigidity tensor and the mass tensor respectively, which are defined as:

$$
\begin{aligned}
& k_{i j}^{*}=\int_{0}^{1} \frac{\partial^{2} w_{i}^{*}}{\partial x^{* 2}} \frac{\partial^{2} w_{j}^{*}}{\partial x^{* 2}} d x^{*} \\
& b_{i j k l}^{*}=\alpha \int_{0}^{1} \frac{\partial w_{i}^{*}}{\partial x^{*}} \frac{\partial w_{j}^{*}}{\partial x^{*}} d x^{*} \int_{0}^{1} \frac{\partial w_{k}^{*}}{\partial x^{*}} \frac{\partial w_{l}^{*}}{\partial x^{*}} d x^{*} \\
& m_{i j}^{*}=\int_{0}^{1} w_{i}^{*} w_{j}^{*} d x^{*}
\end{aligned}
$$

Where $\alpha$ is given by:

$$
\alpha=\frac{h^{2}}{4} \frac{(E S)_{e f f}}{(E I)_{e f f}}
$$

To obtain the nonlinear free response of a clampedclamped FG beam in the neighbourhood of its first resonant frequency, the values of the linear rigidity matrix $k_{i j}{ }^{*}$ and the nonlinear geometrical rigidity tensor $b_{i j k l}{ }^{*}$ have been calculated using the first six normalized symmetric linear beam function $w_{1}{ }^{*}, w_{3},{ }^{*} \ldots, w_{11}{ }^{*}$. The functions $w_{i}^{*}$ have been normalised in such a manner that the obtained mass matrix equals the identity matrix.

\section{Numerical results and discussions}

In the present work, the functionally graded material of the beam is that considered in reference [7]. The top surface of the FG beam is ceramic rich $\left(\mathrm{E}_{\mathrm{c}}=322.03 \mathrm{GPa}\right.$, $\rho_{c}=2370 \mathrm{Kg} / \mathrm{m}^{3}$ ), whereas the bottom surface of the FG beam is metal rich $\left(E_{m}=207.08 \mathrm{GPa}, \rho_{\mathrm{m}}=8166 \mathrm{Kg} / \mathrm{m}^{3}\right)$. In table 1 , It is noted that the first nonlinear frequency ratios $\omega_{n} / \omega_{l}$, calculated in the present work at various vibration amplitudes in the case of an isotropic clampedclamped beam, agree very well with the results obtained in [8], since the percentage error does not exceed $0.3 \%$. However, the solutions given in [7] overestimate the frequencies of the clamped beams, especially for high values of dimensionless amplitude.

Table 1. Non linear to linear Frequency ratios $\omega_{N L}^{*} / \omega_{L}^{*}$ of an isotropic clamped-clamped beam at various vibration amplitudes

\begin{tabular}{cccc}
\hline$W^{a^{*}}\left(x^{*}=0.5\right)$ & Present & Azrar [8] & Fallah [7] \\
\hline 1 & 1.0225 & 1.0221 & 1.0552 \\
2 & 1.0870 & 1.0856 & 1.2056 \\
3 & 1.1869 & 1.1831 & 1.4214 \\
4 & 1.3105 & 1.3064 & 1.6776 \\
\hline
\end{tabular}

In table 2 , the fundamental non linear to linear Frequency ratios $\omega_{n l}^{*} / \omega_{l}^{*}$ of the FG clamped-clamped beam, considered in the present numerical simulation, are 
given and compared with the published results in [7] for various vibration amplitudes.

TABLE 2. Non linear to linear Frequency ratios $\omega_{n l}^{*} / \omega_{l}^{*}$ of a FG Clamped-clamped beam at various amplitudes

\begin{tabular}{|c|c|c|c|c|c|c|}
\hline \multirow[b]{2}{*}{$W^{a^{*}}\left(x^{*}=0.5\right)$} & \multicolumn{2}{|c|}{$\mathrm{n}=0.5$} & \multicolumn{2}{|c|}{$\mathrm{n}=1.0$} & \multicolumn{2}{|c|}{$\mathrm{n}=2.0$} \\
\hline & $\begin{array}{c}\text { presen } \\
\mathrm{t}\end{array}$ & $\begin{array}{c}\text { Falla } \\
\mathrm{h}[12]\end{array}$ & $\begin{array}{c}\text { presen } \\
t\end{array}$ & $\begin{array}{c}\text { Fallah } \\
{[12]}\end{array}$ & present & $\begin{array}{c}\text { Fallah } \\
{[12]}\end{array}$ \\
\hline 0 & 1.000 & 1.000 & 1.000 & 1.00 & 1.000 & 1.00 \\
\hline 1 & 1.0227 & 1.056 & 1.0226 & $\begin{array}{l}0 \\
1.05\end{array}$ & $\begin{array}{l}0 \\
1.021\end{array}$ & $\begin{array}{l}0 \\
1.05\end{array}$ \\
\hline 2 & 1.0878 & 1.210 & 1.0871 & $\begin{array}{l}0 \\
1.20 \\
8\end{array}$ & $\begin{array}{l}1.084 \\
7\end{array}$ & $\begin{array}{l}5 \\
1.20 \\
3\end{array}$ \\
\hline 3 & 1.1874 & 1.429 & 1.1861 & $\begin{array}{l}1.42 \\
6\end{array}$ & $\begin{array}{l}1.181 \\
3\end{array}$ & $\frac{1.41}{7}$ \\
\hline 4 & 1.3131 & 1.689 & 1.3106 & $\begin{array}{l}1.68 \\
5\end{array}$ & $\begin{array}{l}1.303 \\
0\end{array}$ & $\begin{array}{l}1.67 \\
1\end{array}$ \\
\hline 5 & 1.4569 & 1.974 & 1.4536 & $\begin{array}{l}1.96 \\
8\end{array}$ & $\begin{array}{l}1.443 \\
0\end{array}$ & $\begin{array}{l}1.94 \\
9\end{array}$ \\
\hline
\end{tabular}

The same discrepancy is noted in this case, which is mainly due to the inadequate one trial function assumed in [7], which leads to an overestimate of the natural frequencies in comparison with those obtained in the present work in which a multimodal approach is adopted .

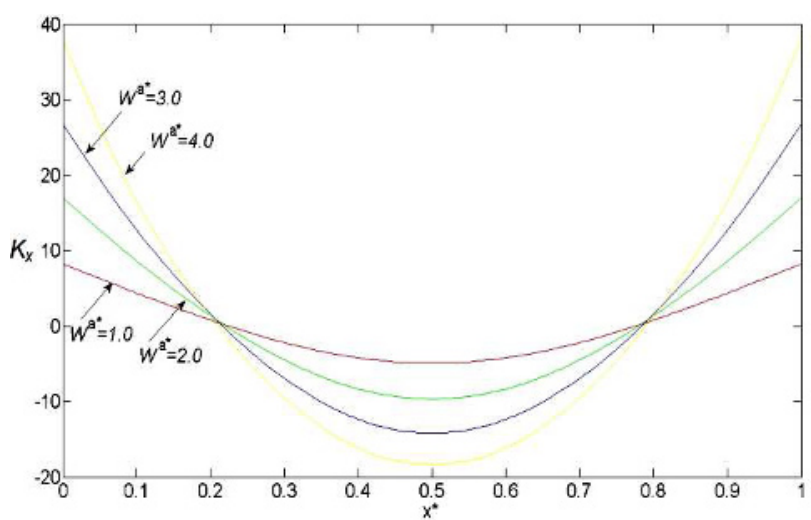

Fig 2. Non-linear free vibration curvatures of a FG clampedclamped beam at amplitudes $\mathrm{Wa}^{*}(\mathrm{x} *=0.5)=1,2,3$ and 4 .

In Fig 2, the curvature distributions associated with the first nonlinear mode of the clamped-clamped FG beam considered here for various values of the maximum vibration amplitudes. It is noted that the curvature is strongly affected by the vibration amplitude, especially at the centre and in the regions of the clamps.

\section{Conclusion}

The present study deals with the problem of geometrically non linear free vibrations of FG beams with immovable ends. Using Hamilton's principle and spectral analysis, and neglecting the axial inertia and damping, an homogenization procedure has been proposed which reduces the problem studied to that of isotropic homogeneous beams with effective bending stiffness and axial stiffness parameters. Using a multimode approach, nonlinear frequency ratios $\omega_{n l} / \omega_{l}$ have been compared to the results published in [7] in the case of isotropic and FG beams. An overestimate of the natural frequencies has been noted in the results given [7], due to the assumed approximate solution adopted. The non-dimensional curvature of the FG clamped beam, associated with the first nonlinear mode, has also been given for different non dimensional amplitudes. It has been clearly shown that the non-linear effect can be significant, especially at the centre and in the regions of the clamps.

The main feature of the present contribution is the fact that the existing analytical solutions, numerical techniques and software developed over the years for the nonlinear analysis of isotropic beams can be easily used for FG beams case, leading to more accurate estimates of the nonlinear dynamic stress, especially near to the clamps, which is a sensitive region in the clamped beam.

\section{References}

1. M. Simsek, Nuclear Engineering and Design, 240 (2010)

2. J. Murín , M. Aminbaghai, V. Kuti, Engineering Structures, 32 (2010)

3. A.E. Alshorbagy, M.A. Eltaher, F.F. Mahmoud , Applied Mathematical Modelling, 35 (2011)

4. S.M.R. Khalili, A.A. Jafari, S.A. Eftekhari, Composite Structures 92 (2010)

5. S. Kapuria, M. Bhattacharyya, A.N. Kumar, Composite Structures 82 (2008)

6. Z. Yu , F. Chu, Journal of Sound and Vibration, 325 (2009)

7. A.Fallah, M.M. Aghdam, European Journal of Mechanics - A/Solids, 30 (2011)

8. L. Azrar, R. Benamar, R.G. White, Journal of Sound and Vibration, 224 (1999) 